\title{
Response scale transfer for visual speed
}

\author{
ALEXANDER SOKOLOV and MARINA PAVLOVA \\ University of Tübingen, Tübingen, Germany \\ and \\ JOHN C. BAIRD \\ Psychological Applications, Newport, New Hampshire, \\ Dartmouth Medical School, Lebanon, New Hampshire, \\ and Dartmouth College, Hanover, New Hampshire
}

\begin{abstract}
By reversing the presentation order and frequency of stimuli between two series of trials, we studied how the category scale for visual speed is transferred across stimulus contexts. Participantsjudged five stimulus speeds, using three categories (slow, moderate, and fast). In Experiment 1, mainly frequent speeds (either low or high) occurred on the initial trials. This manipulation produced divergentpreshift ratings for identical stimuli. Although subsequent reversal of stimulus context resulted in a reversal of scales, the adjustment was incomplete: The postshift ratings did not match the comparable preshift ones. In Experiment 2, mainly infrequent speeds were presented initially. Now the preshift ratings coincided, but higher postshift ratings occurred with the frequent high-speed rather than with the frequent low-speed stimuli. We conclude that with transfer into a new context, the spontaneous adjustment of response scale is determined (1) by the preshift primacy and the postshift frequency effects and (2) by the preshift frequency effect extended to the postshift trials.
\end{abstract}

People and animals perceive, estimate, and act upon things and events occurring in multiple changing contexts. They often accomplish these tasks through generalization, or transfer, of their previous experience. Likewise, in the psychophysics laboratory in which familiarization trials are commonly used prior to experiments proper, a participant's judgment scale established in one situation is transferred or adjusted when applied to another. Whereas in learning studies instructions and explicit feedback typically direct the scale acquisition and generalization, in daily life, situation-linked scale formation and scale transfer across variable contexts often take place spontaneously. In the present study, we examine the spontaneous transfer of a category scale by manipulating the stimulus context (presentation order and frequency of distinct stimuli) between two consecutively presented series of visual speeds.

Research on transfer effects with psychophysicalscales has been confined mostly to studies of the effects of shifts in the range of stimuli (e.g., Ahlström \& Baird, 1989; Algom \& Marks, 1990; Haubensak, 1992; Lockhead, 1992; Marks,

Parts of this work were presented at the 15th Annual Meeting of the International Society for Psychophysics (Tempe, AZ) and at the 22nd European Conference on Visual Perception (Trieste, Italy). M.P. and A.S. are also associated with the Institute of Psychology, Russian Academy of Sciences, Moscow. We thank Niels Birbaumer for providing excellent working facilities and Daniel Algom, Gert Haubensak, and Shuji Mori for valuable comments on an earlier version of the manuscript. Correspondence should be addressed to A. Sokolov, Institute of Psychology, Braunschweig University of Technology, Spielmannstrasse 19, D38106 Braunschweig, Germany (e-mail: a.sikolov@tu-bs.de).
1994; Poulton, 1968, 1979; Thomas, 1993). Any shift in the stimulus range deliberately involves a concomitant change in the stimulus values judged. The main outcome of the studies with moderately differing ranges is that the scales are acquired fairly rapidly (within the initial 3-6 trials), persist over trials, and when transferred into a new situation, change only slightly. This has been documented for a number of tasks, such as category scaling, magnitude estimation, and cross-modality matching (see Ward, 1987). For example, the influence of initial stimulus presentations on loudness scales affected the judgments collected on the following day (Ward \& Lockhead, 1970). After participants judge either lower or upper subranges of differentsized stimuli, they only partly adjust their scales to the whole stimulus range presented subsequently (Haubensak, 1989, 1994). In this study, the ratings of the entire range in the two groups differed out to the 10th postshift series. Comparable findings have been obtained with a variety of stimulus attributes and sensory modalities (e.g., Di Lollo, 1964; Di Lollo \& Casseday, 1965; Johnson, 1949; Parducci, 1954, 1956; Tresselt, 1947). An explicit instruction that participants should modify the original scale in respect to the extended range had little effect (Haubensak, 1989, 1994). These data support Guilford's (1954) view that because the scales are rigidly (without much adjustment) transferred across moderately changing contexts, one need not notify the participants about the impending change.

Response scales appear to more readily adjust to a change in stimulus frequency (e.g., Pollack, 1965); this is also the case when the original range and the values of stimuli are 
preserved. The adjustment can occur even without the observer's attention being explicitly drawn to the change. For example, after judging sets of disproportionately frequent small or large squares, the ratings of stimuli from an oppositely skewed set coincide by the second postshift series (Wedell, 1984). Yet the coincidence of ratings still indicates only a partial scale adjustment: The scales acquired prior to the shift tend to persist. Thus, most of the findings suggest that response scales are fairly stable when transferred into a new stimulus context.

There is, however, some experimental evidence in favor of scale plasticity. Using a few initial trials that were unrepresentative of the overall stimulus distributionin a series, Parducci (1992) found that participants' scales reliably adjusted to the overall set skew. A marked adjustment of the scales within a single series also has been shown by manipulating the initial portion of a presentation sequence and the frequency skew (Sokolov, Pavlova, \& Ehrenstein, 2000). The judgments of identical stimulus speeds depended on a specific combination of the effect of initial stimuli (the primacy effect; Haubensak, 1992, 1994) and the effect of overall stimulus frequency (e.g., Parducci \& Wedell, 1986). The primacy effect more strongly affected the judgments of rarely presented stimuli, whereas frequency affected the judgments of stimuli that occurred mainly on the later trials. When the two effects combined so that the initial trials consisted of many frequent stimuli, identical stimuli from different-skewed sets received divergent ratings. However, when the effects were opposed and mainly infrequent stimuli occurred earlier, identical stimuli received similar ratings. These effects are not accommodated by either Parducci's range-frequency theory (Parducci \& Perrett, 1971; Parducci \& Wedell, 1986) or Haubensak's consistency model (Haubensak, 1992). Each framework accounts for the sole contribution of a single factor, either frequency or serial order of stimuli, respectively, but not for their combination. In addition, because the primacy effect persists over trials and occurs with equal overall stimulus frequencies, the findings are not compatible with Helson's (1964) adaptation-level theory.

An analysis of the response dynamics within a series (Sokolov, Baird, \& Pavlova, 2000) also has demonstrated that judgments specifically depend on a particular combination of the frequency and primacy effects. When, early in a series, participants judge mainly frequent stimuli, the mean rating of a stimulus speed does not change across the trials. When mainly infrequent stimuli occur earlier, however, the mean ratings markedly drift over trials: The ratings for frequent low speeds increase, and those for frequent high speeds decrease. Likewise, as the series unfolds, the probability for a speed to be subsequently assigned to its initial category either does not change or is greatly diminished. The scales, therefore, can exhibit a substantial adjustment to the stimulus context even within a single series of trials.

In the present study, by varying the stimulus context between two successive series of trials, we examined how the presentation order and the frequency of distinct stim- uli determine the transfer of category scales for visual speed. A major limitation of most previous transfer studies has been the confounding effects of several factors (e.g., both the range and the number of distinct stimuli). By contrast, in this study, the stimulus range and the particular stimulus values were kept constant. In the first experiment, the order of stimuli corresponded to their frequency distribution: Mainly frequent speeds occurred on the initial trials. In the second experiment, the orders and skews were opposed - that is, mainly infrequent speeds were presented earlier. Each experiment contained a preshift and a postshift series, whereby a preshift series for one group of participants served as a postshift series for the other. Thus, within a group, we used a complete context reversal from the preshift to the postshift series in respect to the frequency of stimuli and their presentation order.

We hypothesized that the adjustment of the preshift scales to a reversed postshift context would be specifically dependent on the relationship between the order of stimulus presentations and the frequency skew. Namely, with a corresponding order and skew (Experiment 1), the context change should yield a reversal of the relative magnitude of preshift judgments for identical stimuli. In our previous work (Sokolov, Pavlova, \& Ehrenstein, 2000), the primacy effect proved to be long term, persisting for the whole series. An additional goal of Experiment 1, therefore, was to determine whether initial preshift trials affect postshift performance. If this is the case, averaging the data across the preshift and the postshift series should lead to different means for the two groups of observers. In contrast, range-frequency and adaptation-level theories would predict similar group judgments, because the overall frequencies of identical stimuli in the two series are the same.

With the noncorresponding (opposed) order and skew (Experiment 2), the context reversal should result in a postshift separation of the originally coinciding preshift scales. Because the coinciding preshift scales may reflect comparable subjective contexts for judgment, rangefrequency and adaptation-level theories would predict a separation of the scales according to the postshift stimulus frequency. That is, regardless of the postshift presentation order, the higher postshift ratings should obtain with the frequent low speeds, as compared with the frequent high speeds.

\section{EXPERIMENT 1 \\ Scale Transfer With Corresponding Orders and Skews}

A combination of the primacy and the frequency effects generates high-scale ratings with frequent small stimuli and low-scale ratings with frequent large stimuli (Parducci \& Perrett, 1971; Parducci \& Wedell, 1986; Sokolov, Pavlova, \& Ehrenstein, 2000). If these originally acquired scales adjust as the stimulus context is reversed, the high preshift ratings should decrease, whereas the low ratings should increase with the postshift frequent high and low speeds, re- 
spectively. The present experiment was designed to test this conjecture for the stimulus dimension of visual speed.

\section{Method}

Participants. Two different groups of 16 paid observers (8 males and 8 females; mean age, 26.2 years) participated. They all had normal or corrected vision and were naive as to the aim of the study. The participants were run individually.

Stimuli, Stimulus Sets, and Presentation Series. A single light dot $\left(0.15^{\circ}\right.$ in diameter at a viewing distance of $57 \mathrm{~cm}$ with a luminance of $60 \mathrm{~cd} / \mathrm{m}^{2}$ ) traveled horizontally at one of five stimulus speeds ranging from $3 \% \mathrm{sec}$ to $9 \% \mathrm{sec}$. The dot appeared $1.5^{\circ}$ below fixation, centrally within a rectangular aperture $\left(23^{\circ} \times 3^{\circ}\right)$ that was cut in the black cardboard covering the screen of a fast-phosphor 14in. monitor. The speeds were presented in a balanced combination of extent of movement $\left(2^{\circ}, 2.5^{\circ}\right.$, and $\left.3^{\circ}\right)$, duration (seven values ranging from 0.267 to $0.833 \mathrm{sec}$ ), and direction of motion (see Sokolov, Pavlova, \& Ehrenstein, 2000, Table 1, for more details). In order to avoid adaptation, no more than two consecutive trials were run in the same direction (left/right). In the stimulus sets, each speed was generated twice, by using a different combination of duration and extent of motion, and each combination was presented equally often.

Each stimulus set consisted of the five visual speeds $\left(3^{\circ} / \mathrm{sec}\right.$, $4.5 \% \mathrm{sec}, 6 \% \mathrm{sec}, 7.5^{\circ} / \mathrm{sec}$, and $9 \% \mathrm{sec}$ ). The relative proportions of distinct speeds in a set defined the set skew: $20-14-8-4-4$ for a positive skew and 4-4-8-14-20 for a negative skew (Figure 1). For the sake of comparison, the number of stimuli, the set skews, and the number of response categories were chosen to match those used in earlier studies (Parducci \& Wedell, 1986; Sokolov, Pavlova, \& Ehrenstein, 2000). The two different-skewed and ordered sets were presented to different groups of observers. Mainly frequent low- or high-speed stimuli occurred on the initial trials of a series-that is, the order of stimulus presentation corresponded to the set skew. The 50-trial presentation series of stimulus speeds was generated using computer randomization of all stimulus occurrences in a series.

Procedure. On a trial, after a warning tone, a dot appeared within an aperture and started to move. The participants had to verbally judge its speed using three categories (1, slow; 2, moderate; or 3, fast). A head-and-chin rest restricted head movements, while the par-
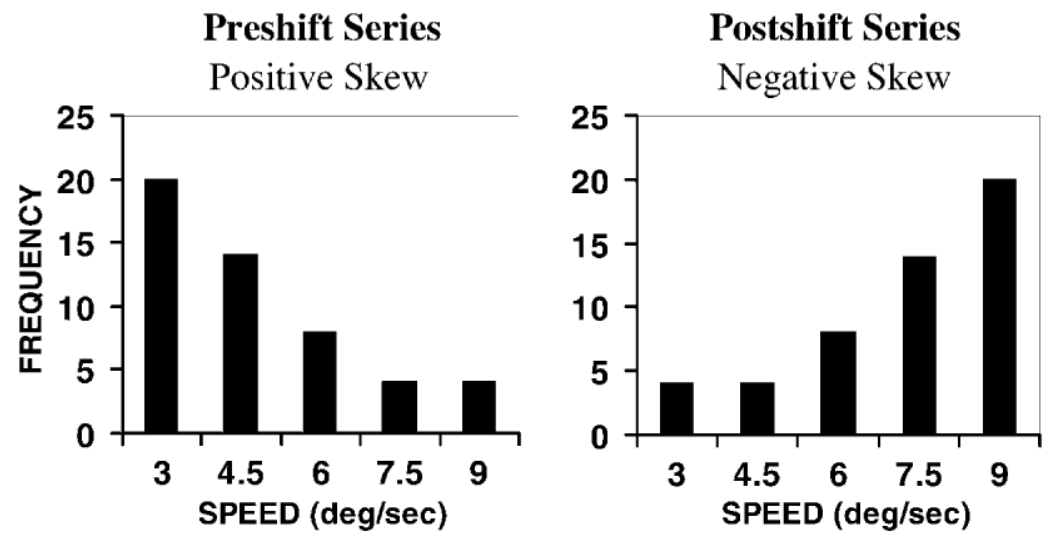

Group A (Expt 1, mainly frequent stimuli occur early)

Group D (Expt 2, mainly infrequent stimuli occur early)
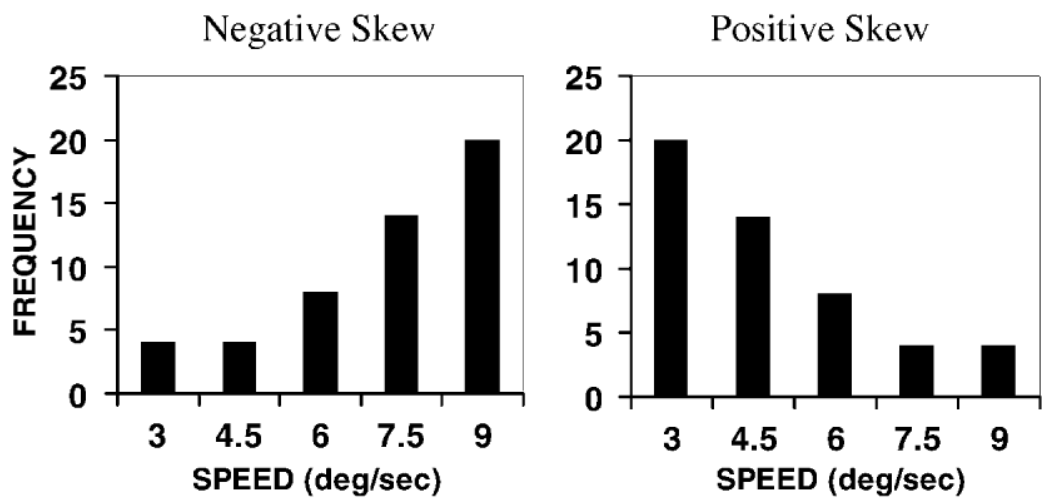

Group B (Expt 1, mainly frequent stimuli occur early) Group C (Expt 2, mainly infrequent stimuli occur early)

Figure 1. Study overview. Frequency distributions for the two stimulus sets of visual speeds used in Experiments 1 and 2. Positively skewed and negatively skewed sets may vary in the presentation order of stimuli. In Experiment 1, mainly frequent stimulus speeds (Groups $A$ and B) and, in Experiment 2, mainly infrequent (low or high) stimulus speeds (Groups D and C) occurred on the initial trials of a series. 


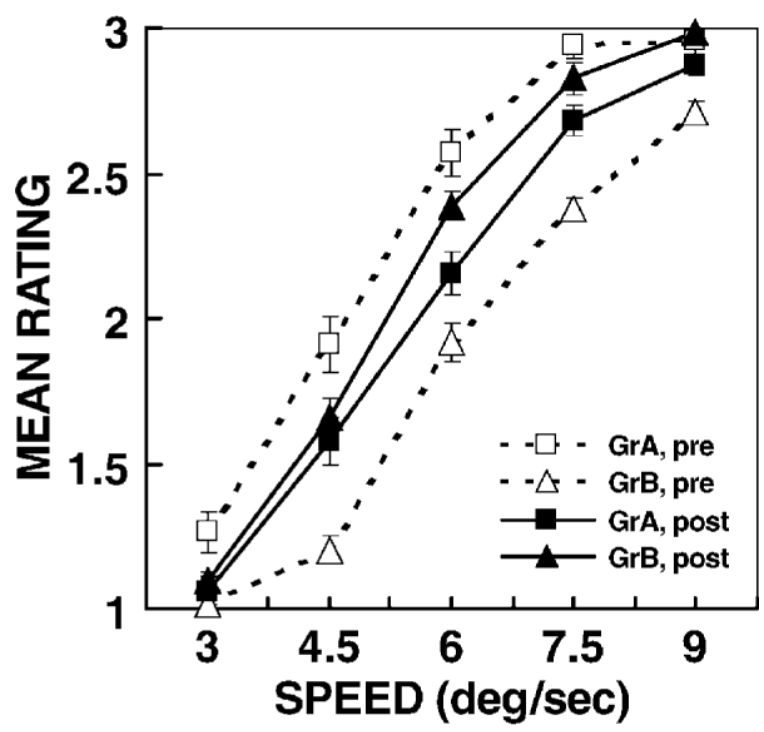

Figure 2. Mean preshift (dashed lines) and postshift (solid lines) ratings for positively and negatively skewed and ordered sets of visual speeds (Experiment 1). Group A judged the preshift frequent low speeds (positive skew, open squares) and the postshift frequent high speeds (negative skew, closed squares), Group $B$ judged the preshift frequent high speeds and the postshift frequent low speeds (open and closed triangles). An experimental point is based on data from 16 participants. Vertical bars show \pm SE.

ticipants kept visually fixating a point at the center of the upper edge of the aperture. They were told about the stochastic combination of extent and duration of motion that produced different speeds and were required to rely strictly on their visual impression of velocity.

The experiment consisted of a preshift and a postshift series of 50 trials; the two series were separated by a 5-10 min break. A preshift series for one group of participants served as a postshift series for the other (Figure 1). The participants were not informed about the shift, nor did they receive any training trials or feedback regarding their performance. It was explicitly stated that there were no right and wrong responses.

\section{Results and Discussion}

Figure 2 shows the preshift (dashed) and postshift (solid curves) judgments of stimulus speeds averaged separately across the participants of Group A (preshift frequent low and postshift frequent high speeds) and of Group B (frequent high speeds and then frequent low speeds).

Inspection of the preshift data indicates that the scale values for the two stimulus sets are clearly different (see also Sokolov, Pavlova, \& Ehrenstein, 2000). With the frequent low-speed stimuli, judgments are displaced upward, as compared with those for the frequent high-speed stimuli. A repeated measures analysis of variance (ANOVA; with group and speed as factors) performed on the mean individual ratings as a dependent measure revealed a significant main effect of group $[F(1,60)=62.106, p<.001]$. The difference in judgments was less at both ends of the scales than in the middle $[F(4,60)=14.801, p<.001$, for the group $\times$ speed interaction].
After the context reversal in the postshift series, in accord with expectations, we observed a pronounced reciprocal adjustment of the preshift scales to a new stimulus context. Identical speeds were estimated lower in Group A and higher in Group B for the postshift series than for the preshift series $[F(1,60)=37.903$ and $63.22, p<.001$, for the main effects of series, respectively]. The adjustment did not proceed uniformly across the speeds; it was smaller for the high-speed stimuli in Group A and for the low-speed stimuli in Group B $[F(4,60)=4.322, p<.004$, and $10.561, p<.001$, for the series $\times$ speed interaction, respectively]. The reversal of the scales resulted in higher postshift ratings obtained with the frequent low speeds (Group B) than with high speeds (Group A): $F(1,60)=$ $6.471, p<.02$, for the main effect of group; see Figure 2 (solid curves). The findings indicate that observers do not rigidly transfer the scales acquired in the preshift series and that the postshift stimulus frequency plays an essential role in the adjustment of a scale. This outcome is in line with the expectations based on range-frequency and adaptation-level theories.

However, the adjustment is incomplete. If it were complete, for identical stimulus sets, the postshift judgments of one group would match the preshift judgments of the other group. Instead, the stimuli from the postshift positively skewed set (with the frequent low speeds) are estimated lower and those from the postshift negatively skewed set (frequent high speeds) are estimated higher than are those in the preshift series (Figure 2). A repeated measures ANOVA, with series and speed as factors, revealed a significant main effect of series $[F(1,60)=5.039$ and $15.923, p<.05$, for positively and for negatively skewed sets, respectively; the corresponding speed $\times$ series interactions were also (barely) significant, $F(4,60)=2.222, p<$ .08 , and $6.294, p<.001]$. Because for identical stimulus sets the judgments made after the shift have been affected by the previous experience with the preshift set, the incomplete scale adjustment is likely to originate from this experience.

This conclusion receives additional support from an analysis of the group judgments averaged across both the preshift and the postshift series. As can be seen in Figure 3, the average judgments of Group A (preshift frequent low speeds and postshift frequent high speeds) exceed those of Group B (preshift frequent high speeds and postshift frequent low speeds). A repeated measures ANOVA, with group and speed as factors, indicated a significant main effect of group $[F(1,124)=7.312, p<.01$; the group $\times$ speed interaction was also significant, $F(4,124)=4.31$, $p<.003]$. The preshift stimulus orders varied between the groups, whereas the overall frequencies of distinct speeds across the two series did not differ between the groups. Therefore, a residual preshift primacy effect appears to be responsible for the difference in the group judgments. This outcome poses a problem for both the adaptation-leveland the range-frequency theories, since they would predict similar average judgments from the groups. At the same 


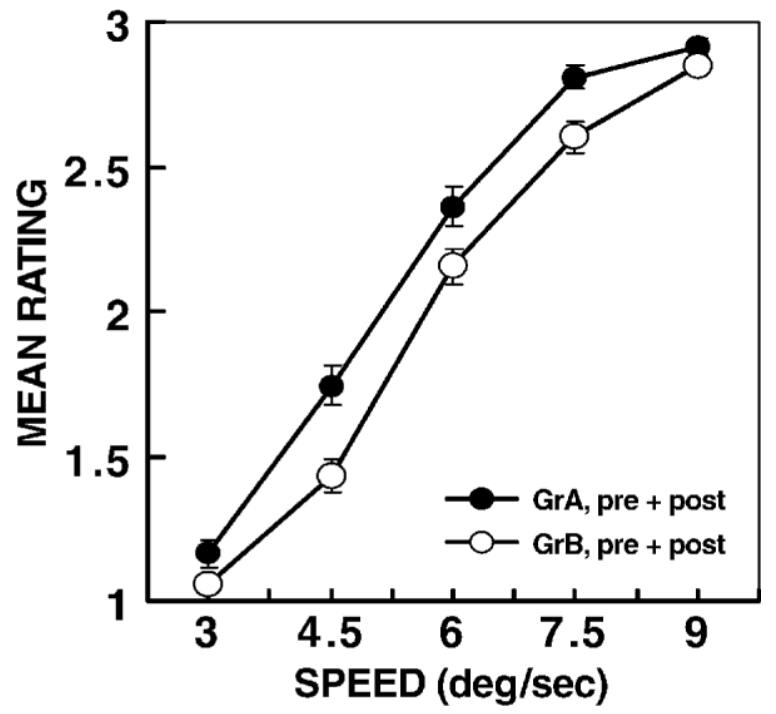

Figure 3. Within-group ratings averaged across both the preshift and the postshift series (Experiment 1). An experimental point is based on data from 16 participants (Group A, closed symbols; Group B, open symbols).

time, these results corroborate the inference from the studies by Haubensak $(1989,1992,1994)$ and by Sokolov, Pavlova, and Ehrenstein (2000) that the primacy effect is long term and may persist well beyond a single series of trials.

The main outcome of Experiment 1 may be summarized as follows. (1) When spontaneously transferred into a reversed context, the divergent preshift scales for visual speed exhibita marked adjustment: The high-scale preshift ratings decrease, and the low-scale ratings increase. The adjustment is primarily determined by the postshift frequency of stimuli. (2) Lack of match between the postshift and the preshift judgments for identical stimulus sets indicates that adjustment is incomplete. This is most likely due to an influence of the remote preshift initial trials (the preshift primacy effect).

In the next experiment, we examined how the context reversal affects the adjustment of identical preshift scales obtained when the primacy and the frequency effects are opposed.

\section{EXPERIMENT 2 Scale Transfer With Opposed Orders and Skews}

When infrequent stimuli dominate the initial trials, the frequency effect that unfolds over the course of the series subsequently compensates for the initial difference in judgments introduced by the primacy effect (Sokolov, Baird, \& Pavlova, 2000; Sokolov, Pavlova, \& Ehrenstein, 2000). As a series proceeds, the occurrences of other, frequent stimuli yield an increase of the initial low ratings (with the infrequent high speeds) and a decrease of high ratings (with the infrequent low speeds). Such reciprocal adjustment of the preshift scales gives rise to their eventual equivalence (Sokolov, Pavlova, \& Ehrenstein, 2000). If in the subsequent series, the response scales continue to adjust to a reversed stimulus context, the postshift ratings should be higher with the infrequent low-speed than with infrequent high-speed stimuli. Experiment 2 was designed to test this prediction.

\section{Method}

Participants. Two different groups of 16 paid observers ( 8 males and 8 females; mean age, 28.5 years) took part in Experiment 2. They did not participate in the previous experiment, had normal or corrected vision, and were naive as to the aim of the study. The participants were run individually.

Stimulus Sets and Presentation Series. The stimulus sets, experimental setup, and procedure of Experiment 2 were the same as those used in Experiment 1. As in Experiment 1, a preshift and a postshift series were employed. Two different groups of participants were exposed to the differently skewed and ordered stimulus sets. A preshift series of one group served as a postshift series for another, so that the stimulus context as to both the set skew and the presentation order of stimuli was reversed from the preshift to the postshift series (Figure 1).

The only difference between the two experiments consisted in the relationship between the presentation order of stimuli in a series and the frequency skew of the sets. Specifically, whereas in Experiment 1 the presentation order corresponded to the set skew (mainly frequent speeds were presented on the initial trials), in Experiment 2 the order and skew were opposed: In both the preshift and the postshift series, mainly infrequent speeds occurred earlier for both groups. For one group (Group C), the preshift series included infrequent low speeds, and the postshift series included infrequent high speeds, presented mostly on the initial trials. The initial preshift trials of the second group (Group D), on the contrary, included mostly infrequent highspeed stimuli and, on the initial postshift trials, mostly infrequent low-speed ones (Figure 1). The respective pseudo-random presentation sequences were generated using a computer randomization of trials in conjunction with a stochastic biasing algorithm developed earlier and described in detail elsewhere (Sokolov, Pavlova, \& Ehrenstein, 2000). Specifically, the relative stimulus frequencies in the two sets from Experiment 1 were first multiplied by five. Each of these (source) distributions was then randomized by a standard computer-based procedure. Finally, from the negatively skewed source sequences, we selected each stimulus speed with the frequency (counting from the start of the sequence) that exactly corresponded to its frequency in the positively skewed set of Experiment 1 . The actual occurrences of the speeds selected from the positively skewed source sequences were equal to those in the negatively skewed sets of Experiment 1.

\section{Results and Discussion}

Figure 4 shows the preshift (dashed) and the postshift (solid curves) judgments averaged separately across the participants of each group. Group C judged the preshift infrequent low and postshift infrequent high speeds, whereas Group D judged the preshift infrequent high and postshift infrequent low speeds. The preshift judgments of the groups did not differ $[F(1,60)=0.263, p>.05$, for the main effect of group].

In the postshift series, however, the judgments with infrequent low speeds were significantly higher than those with infrequent high speeds $[F(1,60)=11.685, p<.004$, 


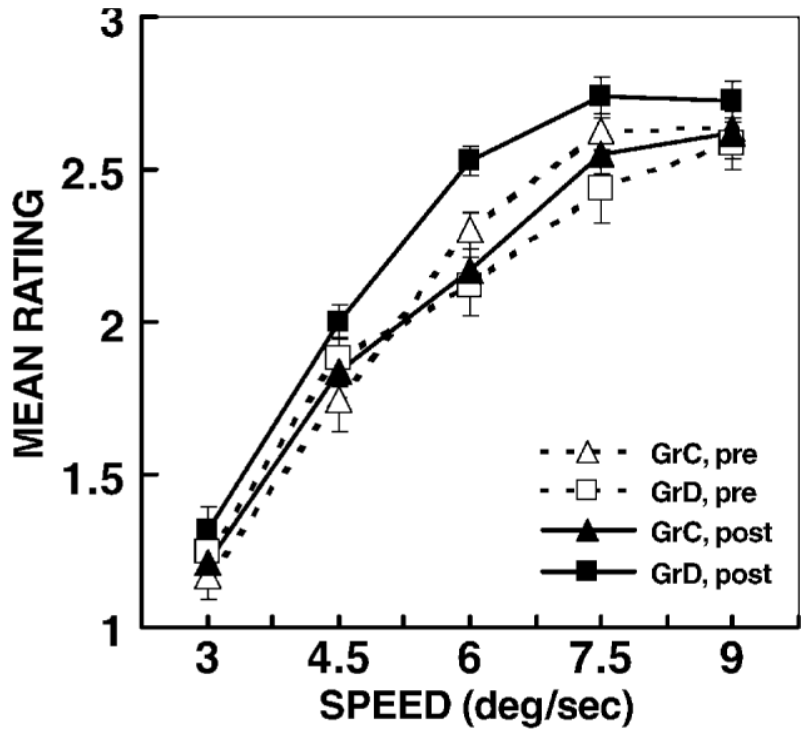

Figure 4. Mean preshift (dashed lines) and postshift (solid lines) ratings for the positively ordered negative-skewed and for the negatively ordered positive-skewed sets of visual speeds (Experiment 2). Group $C$ judged the preshift infrequent low speeds and the postshift infrequent high speeds (open and closed triangles), Group D the preshift infrequent high speeds and the postshift infrequent low speeds (open and closed squares). An experimental point is based on data from 16 participants.

for the main effect of group; the interaction of group and speed was not significant, $F(4,60)=1.466, p>.1]$. Although this result was consistent with our expectations, the higher postshift ratings obtained with infrequent low (i.e., frequent high) speeds are at odds with the postshift judgments observed in Experiment 1, in which the postshift infrequent low speeds yielded lower ratings than did the high speeds (Figure 2, solid curves). The rangefrequency theory (e.g., Parducci \& Perrett, 1971; Parducci \& Wedell, 1986) would also predict lower ratings with the infrequent low-speed stimuli.

Because in Experiment 2 the postshift series started mainly either with infrequent low (Group D) or with high speeds (Group C), a postshift primacy effect (e.g., Haubensak, 1994; Sokolov, Pavlova, \& Ehrenstein, 2000) might account for this striking finding. Prior to the shift, the primacy effect in both groups was reduced by the frequency effect, yielding a match of their preshift judgments (Figure 4 , dashed lines). If a postshift primacy effect were responsible for the difference in ratings, it should be observed in the judgments of the groups averaged across the two (pre- and postshift) series. This was not the case. As can be seen from Figure 5, the average judgments for Group C do not differ from the judgments for Group D $[F(1,124)=1.695, p>.05$, for the main effect of group $]$.

Moreover, if a postshift primacy effect did exist, the judgments averaged across the preshift and the postshift series separately for Group A and for Group B from Experiment 1 should match each other. Actually, the difference in the preshift judgments between Groups A and B resulted from a combination of the primacy and the frequency effects (Figure 2, dashed curves). If the difference in the postshift judgments between the groups was also generated by a combination of these effects, the judgments for the two groups averaged across the preshift and the postshift series would coincide. Yet, as can be seen from the data presented in Figure 3, the average judgments of the groups are clearly distinct (Experiment 1, Results and Discussion section).

An alternative explanation of the postshift effect observed in Experiment 2 assumes that, with the opposed orders and skews, the preshift frequency effect extends to the initial postshift trials. The initial postshift trials mainly consist of the infrequent stimuli that, at the same time, most frequently occurred in the last part of a preceding preshift series. Therefore, because these frequent stimuli elicit a reciprocal adjustment of the preshift response scales (Sokolov, Baird, \& Pavlova, 2000; Sokolov, Pavlova, \& Ehrenstein, 2000), the scales might keep adjusting this way for as long as the occurrences of frequent preshift stimuli prevail in the initial portion of the subsequent postshift series. If this is true, an increase of the preshift ratings produced by the frequent low speeds occurring late in that series should continue into the postshift series, which started mainly with the low-speed stimuli. In turn, a decrease of the initial preshift ratings with the frequent high speeds should also continue into the postshift series starting mainly with the high-speed stimuli. This leads to higher postshift ratings with the infrequent low-speed stimuli than with the high-speed stimuli. The postshift effects found in the present experiment follow this prediction (Figure 4, solid curves).

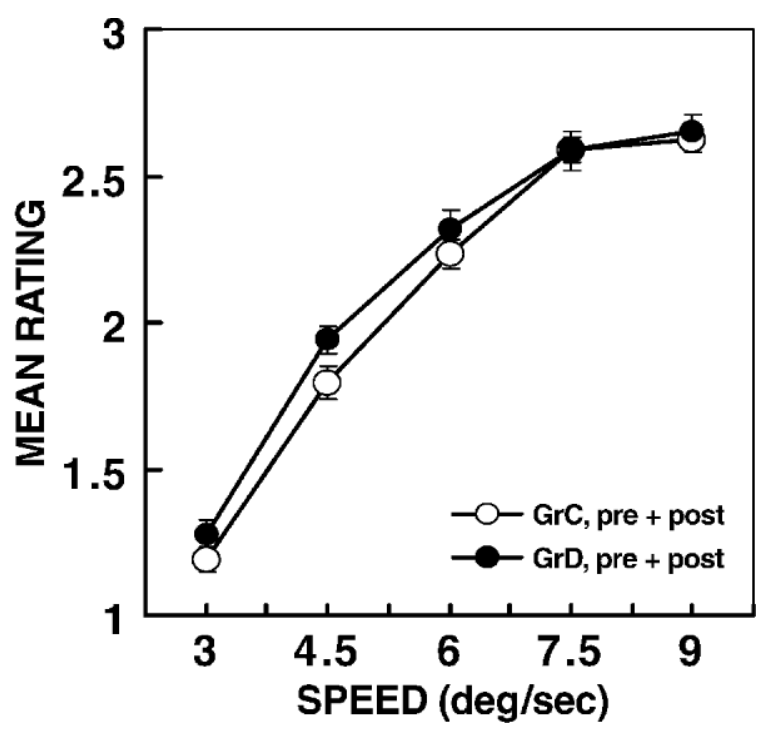

Figure 5. Within-group ratings averaged across both the preshift and the postshift series (Experiment 2). An experimental point is based on data from 16 participants (Group C, open symbols; Group D, closed symbols). 
It is notable that, in contrast with Experiment 1, in Experiment 2 the postshift frequency did not seem to affect the judgments. If this had been the case, the occurrences of frequent low speeds (in Group C) or high speeds (in Group D) accumulated later in the postshift series would have eliminated the difference in the postshift ratings by the groups. This did not occur (see Figure 4). We return to this issue in the General Discussion section. As compared with Experiment 1, both the preshift and the postshift response scales obtained in this experiment are truncated at their lower and upper ends (cf. Figures 2 and 4 ). This finding might be accounted for by the differences in response variability in the two experiments. Recently, we have shown (Sokolov, Baird, \& Pavlova, 2000) that the judgment variability is much greater with the opposed (Experiment 2) than with the corresponding serial order and skew of stimulus sets (Experiment 1). Computer simulations of the judgment process, using the judgment option model of Baird (1997), indicate that the effect of truncation increases with the variability of judgments.

In sum, in Experiment 2, the transfer of coincidingalbeit, generated in different ways - preshift scales into a reversed stimulus context leads to their spontaneous adjustment. In contrast to Experiment 1, however, the observed adjustment runs counter to expectations based purely on the effect of postshift stimulus frequency, nor can it be accounted for by a postshift primacy effect. Instead, the findings suggest that the scale adjustment is determined by the preshift frequency effect that extends to the initial portion of the postshift series.

\section{GENERAL DISCUSSION}

In the present study, we examined the plasticity of category judgments as a function of the statistical context of a stimulus series. Previous data had suggested that despite a change in the stimulus context, people usually keep judging the stimuli mostly according to the initially established scales (e.g., Poulton, 1968, p. 2). In contrast with the early work on transfer effects, which varied the stimulus range together with the number, physical value, or spacing of stimuli in the range (e.g., Ahlström \& Baird, 1989; Haubensak, 1992; Marks, 1994), we used identical sets of visual stimuli throughout the study. We showed that after a reversal of the presentation order and frequency of distinct stimulus speeds between two consecutive series, the response scales readily adjusted to the new stimulus context.

In Experiment 1, in which the order of stimulus presentations in both the preshift and the postshift series corresponded to their frequency of occurrence (i.e., mainly frequent stimuli occurred early in a series), we found that the adjustment of response scale is largely affected by the postshift stimulus frequency. Although the preshift primacy effect prohibited a complete postshift adjustment, the frequent low speeds yielded higher postshift ratings of identical stimuli than did the frequent high speeds (Fig- ure 2, solid curves). These findings extend evidence on the frequency effects established earlier in a betweensubjects design (e.g., Parducci \& Perrett, 1971; Parducci \& Wedell, 1986; Sokolov, Pavlova, \& Ehrenstein, 2000) to transfer situations.

However, in Experiment 2, in which the order of stimuli was opposed to their frequency (i.e., mainly infrequent stimuli occurred earlier), the adjustment of response scale did not exhibit a dependence on the postshift stimulus frequency. In Experiments 1 and 2, we used identical stimulus sets with the same orders and frequencies of stimuli. The only difference between the experiments was that in Experiment 1 the order and frequency corresponded, whereas in Experiment 2 they were opposed. Thus, the postshift stimulus frequency does or does not affect the adjustment of response scale to a changing stimulus context, depending on whether mainly frequent or infrequent stimuli occurred at the outset of a series.

Moreover, in contrast with the postshift findings of Experiment 1 and with the predictions based on rangefrequency theory (e.g., Parducci \& Perrett, 1971), the postshift ratings obtained in Experiment 2 with the frequent low-speed stimuli were lower than those obtained with the frequent high-speed stimuli. This finding might be explained by a preshift frequency effect that operates throughout the series. We refer to it as the extended frequency effect. With the opposed order and frequency, the primacy effect with the infrequent stimuli that prevail in the initial preshift trials first generates divergent judgments of identical speeds (see Sokolov, Baird, \& Pavlova, 2000; Sokolov, Pavlova, \& Ehrenstein, 2000). However, the proportion of stimuli other than those presented early in the preshift series progressively increases by the end of the series. This leads to rapid reciprocal adjustment of the early scales and to reduction of the primacy effect and results in the eventual match of the preshift scales (Figure 4, dashed curves). Immediately after the context reversal, the occurrences of these stimuli (frequent in the preceding preshift series, but infrequent in the postshift series) still continue to accumulate, exerting a primacy-like effect of preshift stimulus frequency on the postshift ratings, or in other words, an extended frequency effect (Figure 4, solid curves).

The frequent low-speed stimuli from the postshift phase do not seem to exert any effect on judgments, most likely because their relative proportion is small, as compared with the pre- and postshift frequency of other stimuli. As has been recently demonstrated (Sokolov, Pavlova, \& Ehrenstein, 2000), an eventual influence of these stimuli in the initial preshift trials is subsequently canceled out by progressively accumulating occurrences of other stimuli by the end of the preshift phase. This account might appear consonant with the proposal made in an elaborated (retrieval-consistency) version of range-frequency theory by Parducci and Wedell (1986). In this version, it is suggested that only the frequencies of most recent stimuli exert a prominent effect on judgments. If this were true, 
however, the effect of preshift frequencies extended into the outset of the postshift phase would be subsequently overrun as the frequency of other stimuli increases in the remainder of the postshift series. This should yield either similar postshift judgments or greater judgments with the frequent low speeds than with the high speeds. Yet the data from Figure 4 (solid curves) indicate that the greater judgments were obtained with the postshift frequent highspeed stimuli.

The present results therefore suggest a reciprocal adjustment of the response scale to the reversed presentation order of stimuli and their frequency across the preshift and postshift series. The spontaneous adjustment is determined either (1) by both the preshift primacy and the postshift frequency effects or (2) solely by the preshift frequency effect that extends into the postshift trials. The outcome of adjustment is dependent on the relationship of the serial order and the frequency of stimuli in the sets.

Overall, the data a challenge for the major existing theoretical accounts of the judgment process, because each deals with only a single aspect of the findings. The consistency model encounters difficulty when explaining the transfer effects associated with the stimulus frequency in the pre- and postshift series, whereas the range-frequency and adaptation-level theories have trouble explaining the long-lasting influence of remote preshift stimulus presentations (the primacy effect). A new theoretical framework is required that will properly reconcile the combined effects of the two experimental variables.

The findings have several implications for studies in which an adjustment of the response scales can be expected. First, they emphasize the importance of sequential or stepwise data analyses (e.g., Baird, 1997; Huettel \& Lockhead, 1999; Sokolov, Baird, \& Pavlova, 2000), as opposed to the prevailing tendency to present average, summary measures of performance (as characterized by the original work of Stevens, 1975). In the present study, for example, it would be difficult to reconcile the contrasting postshift data obtained in the two experiments without considering separately the nature of the pre- and the postshift series. Likewise, in Experiment 2, the difference between the groups in the postshift series (Figure 4, solid curves) was masked in the group judgments averaged across both the preshift and the postshift series (Figure 5).

Second, the preshift series of this study may be considered as a kind of familiarization phase commonly used prior to the start of psychophysical experiments. Because our data indicate that the preshift series can largely affect later performance, it is important to monitor the presentation order and frequency of the stimuli used in the familiarization phase of a study. A related procedural point is that the scales to be generalized in generalization studies should be comparable in their origin, since the way in which the response scales are acquired can specifically alter subsequent stimulus categorization.

Generally, a psychophysical scale is characterized as a transformation on a set of stimulus values that maps onto subjective values. In the present study, the judgment curves for identical speeds in one condition (group, series, etc.) did not match the respective curves in another condition (cf., e.g., Figures 2 and 4). This suggests that a particular mapping is not preserved across variable stimulus contexts. Rather, the scale depends on the contextual statistics in such a way that the context can modify the very form of the scale. Our findings therefore demand an elaboration of psychophysical theory concerning the contextual dependencies related to the presentation order and the frequency of stimuli.

\section{REFERENCES}

Ahlström, R., \& BAIRd, J. C. (1989). Shift in stimulus range and the exponent of the power function for loudness. Perception \& Psychophysics, 46, 603-607.

Algom, D., \& MARKs, L. E. (1990). Range and regression, loudness scales, and loudness processing: Toward a context-bound psychophysics. Journal of Experimental Psychology: Human Perception \& Performance, 16, 706-727.

BAIRD, J. C. (1997). Sensation and judgment: Complementarity theory of psychophysics. Mahwah, NJ: Erlbaum.

Di Lollo, V. (1964). Contrast effects in the judgment of lifted weights. Journal of Experimental Psychology, 68, 383-387.

Di Lollo, V., \& Casseday, J. H. (1965). Graded contrast effects in the judgment of lifted weights. Journal of Experimental Psychology, 70, 234-235.

Guilford, J. P. (1954). Psychometric methods (2nd ed.). New York: McGraw-Hill.

Haubensak, G. (1989). Primacy-Effekte in psychophysischen Urteilen [Primacy effects in psychophysical judgments]. Zeitschrift für experimentelle und angewandte Psychologie, 36, 42-56.

HaubensaK, G. (1992). The consistency model: A process model for absolute judgments. Journal of Experimental Psychology: Human Perception \& Performance, 18, 303-309.

HaUbENSAK, G. (1994). Wie entsteht der Häufigkeitseffekt in absoluten Urteilen: Ein Beitrag zur Theorie des Urteilens [How does the frequency effect in category ratings arise? A contribution to rating theory]. Zeitschrift für experimentelle und angewandte Psychologie, 41, 378-397.

Helson, H. (1964). Adaptation-level theory. New York: Harper \& Row. Huettel, S. A. \& Lockhead, G. R. (1999). Range effects of an irrelevant dimension on classification. Perception \& Psychophysics, 61, 1624-1645.

Johnson, D. M. (1949). Learning function for a change in the scale of judgment. Journal of Experimental Psychology, 39, 851-860.

LockHEAD, G. R. (1992). Psychophysical scaling: Judgments of attributes or objects? Behavioral \& Brain Sciences, 15, 543-601.

Marks, L. E. (1994). "Recalibrating" the auditory system: The perception of loudness. Journal of Experimental Psychology: Human Perception \& Performance, 20, 382-396.

Parducci, A. (1954). Learning variables in the judgment of single stimuli. Journal of Experimental Psychology, 48, 24-30.

Parducci, A. (1956). Direction of shift in the judgment of single stimuli. Journal of Experimental Psychology, 51, 169-178.

Parducci, A. (1992). Comment on Haubensak's associative theory of judgment. Journal of Experimental Psychology: Human Perception \& Performance, 18, 310-313.

Parducci, A., \& Perrett, L. F. (1971). Category rating scales: Effects of relative spacing and frequency. Journal of Experimental Psychology Monographs, 89, 427-452.

Parducci, A., \& Wedell, D. H. (1986). The category effect with rating scales: Number of categories, number of stimuli, and method of presentation. Journal of Experimental Psychology: Human Perception \& Performance, 12, 496-516.

PolLACK, I. (1965). Neutralization of stimulus bias in the ratings of gray. Journal of Experimental Psychology, 69, 564-578.

Poulton, E. C. (1968). The new psychophysics: Six models for magnitude estimation. Psychological Bulletin, 69, 1-19. 
Poulton, E. C. (1979). Models for biases in judging sensory magnitudes. Psychological Bulletin, 86, 777-803.

Sokolov, A., Baird, J. C., \& Pavlova, M. (2000). When first love fades: What makes judgment scales drift over trials. In C. Bonnet (Ed.), Fechner Day 2000 (pp. 333-338). Strasbourg: International Society for Psychophysics.

Sokolov, A., Pavlova, M., \& Ehrenstein, W. H. (2000). Primacy and frequency effects in absolute judgments of visual velocity. Perception \& Psychophysics, 62, 998-1007.

Stevens, S. S. (Ed.) (1975). Psychophysics: Introduction to its perceptual, neural, and social prospects. New York: Wiley.

Thомаs, D. (1993). A model for adaptation-level effects on stimulus generalization. Psychological Review, 100, 658-673.

Tresselt, M. E. (1947). The influence of amount of practice upon the formation of a new scale of judgment. Journal of Experimental Psychology, 37, 251-260.

WARD, L. M. (1987). Remembrance of sounds past: Memory and psychophysical scaling. Journal of Experimental Psychology: Human Perception \& Performance, 13, 216-227.

WARD, L. M., \& LockHEAD, G. R. (1970). Sequential effects and memory in category judgments. Journal of Experimental Psychology, 84, 27-34.

WedELL, D. H. (1984). A process model for psychophysical judgment. Dissertation Abstracts International, 45, 3102-B. (University Microfilms International No. 8428589)

(Manuscript received February 2, 2001; revision accepted for publication August 13, 2001.) 